\title{
Peak or plateau maximal inspiratory mouth pressure: which is best?
}

\author{
W. Windisch*, E. Hennings*, S. Sorichter*, H. Hamm*, C.P. Criée
}

Peak or plateau maximal inspiratory mouth pressure: which is best? W. Windisch, E. Hennings, S. Sorichter, H. Hamm, C.P. Criée. C) ERS Journals Ltd 2004.

ABSTRACT: There is no clear evidence as to how maximal inspiratory mouth pressure $(P I, m a x)$ should be measured, although plateau pressures sustained for $1 \mathrm{~s}$ and measured at residual volume (RV) are usually recommended.

Peak and plateau $P I$,max were measured at $R V$ and at functional residual capacity (FRC) in 533 healthy subjects (aged 10-90 yrs) in order to comparably test all PI,max measurements for their predictors, reproducibility and normal values.

Plateau pressures accounted for $82.0-86.3 \%$ of peak pressures. Peak and plateau pressures measured at FRC accounted for $84.3-90.5 \%$ of pressures at $\mathrm{RV}$, and were highly correlated. Age was negatively predictive and weight and body mass index positively predictive of $P I$,max, but regression parameters were low. All $P$ I,max measurements were comparable when calculating regression parameters, between-subject variability and reproducibility.

In conclusion, peak and plateau maximal inspiratory mouth pressure are comparably useful for the assessment of inspiratory muscle strength and can be reliably measured at functional residual capacity and at residual volume. Regression equations are of low impact in predicting normal values due to the weak influence of demographic and anthropometric factors and to the high unexplained between-subject-variability. Agerelated 5th percentiles can indicate the lower limit of the normal range.

Eur Respir J 2004; 23: 708-713.
*Dept of Pneumology, University Hospital Freiburg, Freiburg, "North Sea Clinic Westerland, Westerland, and "Dept of Pneumology, Respiratory Intensive Care Unit and Sleep Laboratory, Evangelical Hospital GöttingenWeende Incorporated Society, Bovenden-Lenglern, Germany.

Correspondence: W. Windisch, Dept of Pneumology, University Hospital Freiburg, Killianstraße 5, D-79106 Freiburg, Germany.

Fax: 497612703704

E-mail: windisch@med1.ukl.uni-freiburg.de

Keywords: Muscle strength

normal values

reproducibility

respiratory failure

respiratory muscles

respiratory pressures

Received: December 82003

Accepted: December 222003
Noninvasive measurement of maximal inspiratory mouth pressure $(P I, \max )$ is the simplest and most widely used specific diagnostic test for the quantification of inspiratory muscle strength, thus facilitating the diagnosis of inspiratory muscle weakness $[1,2]$. Several studies aimed at assessing so-called normal values have been conducted in the past in order to facilitate interpretation of $P I$, max measurements in patients with impaired respiratory muscle function [3-16].

Both absolute mean normal values and regression equations for calculating normal values differ significantly among these studies. Therefore, a standardised approach to testing performance and measurement was proposed in a recent American Thoracic Society (ATS)/European Respiratory Society (ERS) statement on respiratory muscle testing devised by an expert panel [1]. Although not evidence-based, this statement suggested that plateau pressures sustained for $1 \mathrm{~s}$ are to be preferred over peak pressures $(P \mathrm{I}$,peak), and that $P$ I,max should be measured at or close to the residual volume (RV) rather than at the functional residual capacity (FRC) [1].

To date, no study has clearly demonstrated the benefits of plateau pressures and pressures measured at RV. Conversely, $P I$,peak might be easier to calculate than plateau pressures [1], and pressures measured at FRC reflect inspiratory muscle strength more exactly than pressures measured at RV, which are overestimated due to the additional passive elastic recoil of the respiratory system.

Therefore, the aim of the present study was to test the hypothesis that $P \mathrm{I}$,peak compared to plateau pressures and pressures measured at FRC compared to pressures measured at $\mathrm{RV}$, respectively, are comparably or even more useful in the assessment of inspiratory muscle strength. Thus the study aimed to provide normal values for all $P \mathrm{I}$,max measurements.

\section{Methods}

The study protocol was approved by the Agency of Ethics of Albert-Ludwig University, Freiburg, Germany, and was performed in accordance with the ethical standards laid down in the Declaration of Helsinki. Informed written consent was obtained from all participants.

\section{Study population}

Recruitment of participants was performed at six different locations in order to avoid enrolment of selected participants. It was thereby ensured that participants with a wide range of sociodemographic characteristics would be enrolled. Both urban (city of 200,000 inhabitants) and rural populations were recruited, with measurements being made at the University Hospital of Freiburg, Freiburg, Germany (employees and visitors) and at different registered associations and public facilities (i.e. places where people meet in clubs, institutions, societies, etc.). Since health status is positively associated with $P \mathrm{I}$, max [14], only healthy participants were studied. Therefore, stringent exclusion criteria were established: pre-existing lung diseases and airway diseases, chest wall deformities, neuromuscular diseases, neurological deficits (stroke, multiple sclerosis, hemiplegia, parkinsonism and extrapyramidal disease), coronary heart 
disease, congestive heart failure, endocrine disturbances, respiratory infection, malignant diseases, following thoracic or abdominal surgery, and medication (systemic or inhaled glucocorticoids, mineralocorticoids, central nervous system stimulants, theophylline, hypnotic or sedative agents, muscle relaxants and hormones). Spirometry was performed prior to $P I$,max determination in order to exclude participants with reduced lung function, as defined by a forced expiratory volume in one second (FEV1) or inspiratory vital capacity (IVC) of $<85 \%$ of the predicted value, according to the official statement of the ERS [17].

\section{Spirometric and maximal inspiratory mouth pressure measurements}

Both spirometric and $P \mathrm{I}$,max measurements were performed using transportable apparatus connected to a computer system (ZAN 100; ZAN®, Oberthulba, Germany). For measurement of $P I$, max, a shutter with a magnetic catch piston was used to completely occlude the external airway for $2.0 \mathrm{~s}$. The pressure transducer was interfaced with the computer, allowing visualisation of the pressure/time curves. Calibration of the system was performed daily prior to use. $P I$,max measurements were performed only by one specialised person. Participants were instructed to exert maximal inspiratory effort after slow exhalation and were encouraged by the investigator to "suck harder" during each PI,max manoeuvre. All $P$ I,max were measured with the participant in a seated position and wearing a nose clip. A flanged mouthpiece was used with a small leak ( $2 \mathrm{~mm}$ internal diameter) to prevent glottic closure during the manoeuvre [1].

All PI,max were measured at both RV and FRC, and both $P$ I,peak and plateau pressure were recorded during each $P$ I,max manoeuvre. The lung volume from which the manoeuvre was initiated was controlled spirometrically. First, quiet breathing with consistent pressure curves was confirmed by visualisation of the pressure/time curves on the monitor prior to the $P I$, max manoeuvre. The elapsed time between successive manoeuvres ranged $30-120 \mathrm{~s}$. The volume at end-expiration during the last breath prior to the $P \mathrm{I}, \max$ manoeuvre while breathing quietly was regarded as $0 \mathrm{~L}$ (FRC). The difference between the lung volume from which the manoeuvre was initiated and $0 \mathrm{~L}$ was calculated for each manoeuvre. Accordingly, a high difference in lung volumes was expected during RV manoeuvres, but a difference close to $0 \mathrm{~L}$ during FRC manoeuvres.

Plateau pressures were defined as pressures that could be sustained for $0.5(P \mathrm{I}, \max , 0.5)$ and $1.0 \mathrm{~s}(P \mathrm{I}, \max , 1.0)$ at the minimal value of the maximal pressure window over 0.5 and $1.0 \mathrm{~s}$, respectively. In addition, the pressure $100 \mathrm{~ms}$ after the start of maximal inspiratory effort $(P I, \max , 0.1)$ was recorded from the same pressure/time curve.

\section{Study design}

The height and weight of all participants were measured. Sitting height was calculated as has been described previously [14]. Body mass index (BMI) was also calculated. After spirometry, at least seven $P \mathrm{I}$, max trials were completed by each participant at both RV and FRC and in random order, with the highest pressure obtained being selected. A further three trials were performed if the second largest PI,peak was $>10 \%$ lower than the largest $P$ I,peak. In addition, the measurements were repeated in 25 females and 25 males 1-4 weeks after the initial measurement and using the same study protocol to assess the reproducibility of PI,max measurements.

\section{Statistical analysis}

Significance was assumed at a $p$-value of $<0.05$. Descriptive data are presented as mean \pm SD after testing for normal distribution. Correlation analysis was performed using Pearson product-moment correlation. Stepwise multiple regression models for each sex were constructed with PI,peak, $P \mathrm{I}, \max , 0.5, P \mathrm{I}, \max , 1.0$ and $P \mathrm{I}, \max , 0.1$ as dependent variables. Age, height, sitting height, BMI and weight were used as independent variables. Regression parameters were calculated and compared to values obtained from the literature. In addition, analysis of covariance was performed. Since all $P \mathrm{I}$,max were measured at both FRC and $\mathrm{RV}$, the difference between values at both levels of measurement was calculated using repeated measures analysis of variance. For $P$ I,peak and $P \mathrm{I}$,max, 1.0 measured at $\mathrm{RV}$, the 5th and 50th (median) percentiles were calculated for age group and sex. Finally, in the 50 participants in whom the reproducibility of $P \mathrm{I}$, max was also studied, the coefficient of repeatability (CR) was calculated using the methods of BLAND and ALTMAN [18]. All $P I$ max were measured as negative with respect to ambient pressure. However, all $P \mathrm{I}$,max were reported as positive values in order to avoid confusion during the interpretation and discussion of the data, since positive values have been given in nearly all former studies.

\section{Results}

A total of 533 healthy participants completed the study protocol (table 1). No adverse effects or complications were observed during measurement of PI,max. Mean IVC was

Table 1.-Demographic data

\begin{tabular}{|c|c|c|c|}
\hline & Males & Females & All subjects \\
\hline Subjects $n$ & 229 & 304 & 533 \\
\hline Age yrs & $34.3 \pm 14.8(11-80)$ & $38.5 \pm 15.2(10-90)$ & $36.4 \pm 14.8(10-90)$ \\
\hline Height $\mathrm{cm}$ & $179.5 \pm 7.7(143-199)$ & $166.4 \pm 7.0(147-188)$ & $172.0 \pm 9.8(143-199)$ \\
\hline Sitting height $\mathrm{cm}$ & $91.4 \pm 4.2(75-102)$ & $86.7 \pm 3.3(77-96)$ & $88.7 \pm 5.1(75-102)$ \\
\hline Weight kg & $77.9 \pm 11.2(32-115)$ & $66.0 \pm 10.9(38-109)$ & $71.1 \pm 12.5(32-115)$ \\
\hline $\mathrm{BMI} \mathrm{kg} \cdot \mathrm{m}^{-2}$ & $24.2 \pm 3.1(15.6-34.0)$ & $23.9 \pm 4.1(16.4-39.6)$ & $24.0 \pm 3.7(15.6-39.6)$ \\
\hline Cumulative smoking pack-yrs & $4.0 \pm 9.9(0-105)$ & $2.6 \pm 5.6(0-35)$ & $3.2 \pm 7.8(0-105)$ \\
\hline Active smokers $\mathrm{n}$ & 120 & 197 & 317 \\
\hline Former smokers $\mathrm{n}$ & 48 & 36 & 84 \\
\hline Never smokers $\mathrm{n}$ & 60 & 68 & 128 \\
\hline
\end{tabular}

Data are presented as mean \pm SD (range). Data regarding smoker status are not available for four participants. BMI: body mass index. 
Table 2.-Peak (Pl,peak) and plateau maximal inspiratory mouth pressures measured at residual volume (RV) and functional residual capacity (FRC)

\begin{tabular}{|c|c|c|c|c|}
\hline & \multicolumn{2}{|c|}{ Males } & \multicolumn{2}{|c|}{ Females } \\
\hline & RV & FRC & RV & FRC \\
\hline Subjects $\mathrm{n}$ & \multicolumn{2}{|c|}{229} & \multicolumn{2}{|c|}{304} \\
\hline$P \mathrm{I}$, peak $\mathrm{kPa}$ & $12.4 \pm 3.6$ & $11.0 \pm 3.4$ & $8.9 \pm 3.4$ & $7.5 \pm 3.0$ \\
\hline$P I, \max , 0.5 \mathrm{kPa}$ & $11.3 \pm 3.4$ & $10.2 \pm 3.2$ & $8.0 \pm 3.3$ & $6.8 \pm 2.9$ \\
\hline$P I, \max , 1.0 \mathrm{kPa}$ & $10.5 \pm 3.4$ & $9.5 \pm 3.1$ & $7.3 \pm 3.1$ & $6.2 \pm 2.7$ \\
\hline$P \mathrm{I}, \max , 0.1 \mathrm{kPa}$ & $4.8 \pm 2.2$ & $2.9 \pm 1.5$ & $3.3 \pm 1.7$ & $1.9 \pm 0.9$ \\
\hline
\end{tabular}

Data are presented as mean \pm SD. PI,max,0.5: plateau maximal inspiratory mouth pressure sustained for $0.5 \mathrm{~s}$; $P \mathrm{I}, \max , 1.0$ : plateau maximal inspiratory mouth pressure sustained for $1.0 \mathrm{~s} ; P \mathrm{I}, \max , 0.1$ : maximal inspiratory mouth pressure $100 \mathrm{~ms}$ after start of maximal inspiratory manoeuvre.

$4.4 \pm 1.1 \mathrm{~L}$ (range 1.9-7.2 L), i.e. $103.9 \pm 11.3(85.0-157.4) \%$ pred. Mean FEV1 was $3.5 \pm 0.8 \mathrm{~L}$ (range $1.4-5.8 \mathrm{~L}$ ), i.e. $99.8 \pm 10.6(85.0-154.7) \%$ pred.

When PI,max was measured after maximal exhalation, the lung volume at which the $P \mathrm{I}, \max$ manoeuvres were started averaged $-0.70 \pm 0.39$ (females) and $-1.03 \pm 0.48 \mathrm{~L}$ (males) compared to FRC during quiet breathing. This indicates further exhalation from FRC prior to the $P \mathrm{I}$,max manoeuvre (RV). In contrast, the lung volume after normal exhalation during measurements at FRC averaged $0.02 \pm 0.16$ (females) and $-0.04 \pm 0.20 \mathrm{~L}$ (males), indicating that the $P \mathrm{I}$, $\max$ manoeuvres were indeed performed very close to FRC.

$P$ I, peak were higher than plateau pressures $(\mathrm{p}<0.001)$, but the $P \mathrm{I}$,max, 0.1 was lower than $P \mathrm{I}$,peak and plateau pressures $(\mathrm{p}<0.001)$ (table 2). The mean $P$ I,max in females accounted for $68.8-71.8 \%$ of that in males when measured at RV, and for $65.3-68.2 \%$ when measured at FRC $(\mathrm{p}<0.0001)$. Mean $P$ I,max measured at FRC amounted to $84.3-85.0 \%$ in females and $88.7-90.5 \%$ in males of that measured at RV (PI,peak, $P$ I,max,0.5, $\quad P$ I,max,1.0) $\quad(\mathrm{p}<0.0001)$. The mean $P$ I,max, 0.1 measured at FRC was 57.6 (females) and 60.4\% (males) of that measured at RV. There was a close correlation between $P$ I,peak and plateau pressures, but $P \mathrm{I}, \max , 0.1$ correlated more weakly (table 3 ). The 5th and 50th (median) percentiles for $P \mathrm{I}$,peak and $P \mathrm{I}$,max,1.0 measured at $\mathrm{RV}$ are given in table 4 .

The highest $r^{2}$ (multiple regression) were identified for age when calculated separately. In females, $\mathrm{r}^{2}$ was 0.13 (PI,max, 1.0 at $\mathrm{RV}$ ) and 0.14 (PI,max,1.0 at FRC), indicating that age accounted for 13 and $14 \%$, respectively, of the total variance of $P \mathrm{I}$,max,1.0 in females. In males, $\mathrm{r}^{2}$ was $0.02(P \mathrm{I}, \mathrm{max}, 1.0$ at $\mathrm{RV}$ and FRC), indicating that age accounted for $2 \%$ of the total variance of $P \mathrm{I}, \max , 1.0$ in males. Comparable results were calculated for $P \mathrm{I}$,peak, $P \mathrm{I}, \max , 0.5$ and $P \mathrm{I}, \max , 0.1$, with the highest $\mathrm{r}^{2}$ of 0.16 calculated for age in females ( $P$ I,peak at RV).
Table 3. - Pearson correlation coefficients for peak ( $P$ l,peak) and plateau maximal inspiratory mouth pressures measured at residual volume (RV) and functional residual capacity (FRC)

\begin{tabular}{ccccc}
\hline & Variable & PI,max,0.5 & PI,max,1.0 & $P$ I,max,0.1 \\
\hline RV & & & & \\
Females & PI,peak & $0.97^{\#}$ & $0.94^{\#}$ & $0.52^{\#}$ \\
& PI,max,0.5 & & $0.98^{\#}$ & $0.50^{\#}$ \\
Males & PI,max,1.0 & & & $0.51^{\#}$ \\
& PI,peak & $0.97^{\#}$ & $0.94^{\#}$ & $0.56^{\#}$ \\
& PI,max,0.5 & & $0.97^{\#}$ & $0.54^{\#}$ \\
FRC & PI,max,1.0 & & & $0.53^{\#}$ \\
Females & PI,peak & $0.98^{\#}$ & $0.94^{\#}$ & $0.51^{\#}$ \\
& PI,max,0.5 & & $0.97^{\#}$ & $0.51^{\#}$ \\
Males & PI,max,1.0 & & & $0.50^{\#}$ \\
& PI,peak & $0.96^{\#}$ & $0.93^{\#}$ & $0.48^{\#}$ \\
& PI,max,0.5 & & $0.97^{\#}$ & $0.48^{\#}$ \\
& PI,max,1.0 & & & $0.53^{\#}$ \\
\hline
\end{tabular}

Data represent 533 subjects (304 females and 229 males). PI,max,0.5: plateau maximal inspiratory mouth pressure sustained for $0.5 \mathrm{~s}$; PI,max,1.0: plateau maximal inspiratory mouth pressure sustained for $1.0 \mathrm{~s} ;$ PI,max,0.1: maximal inspiratory mouth pressure $100 \mathrm{~ms}$ after start of maximal inspiratory manoeuvre. ${ }^{\#}: \mathrm{p}<0.0001$

The $\mathrm{r}^{2}$ calculated for weight, height, BMI and sitting height were even lower those calculated for age for all $P$ I,max ranging $0-0.04$. The interaction of age and weight, height, BMI or sitting height resulted in nonessential higher $\mathrm{r}^{2}$.

In the final regression model, height and sitting height were not significant predictors of $P I$,max. In contrast, age was negatively predictive, but weight and BMI were positively predictive for all $P \mathrm{I}$,max measurements. There was no significant difference between $P \mathrm{I}$,peak, $P \mathrm{I}$,max, $0.5, P \mathrm{I}, \max , 1.0$ and $P$ I,max,0.1 in terms of their predictors. The regression parameters for $P \mathrm{I}$,peak and $P \mathrm{I}, \max , 1.0$ are given in table 5. The age-related decline in $P I$, max was stronger in females than in males, expressed by more negative regression parameters $(\mathrm{p}<0.05)$ (table 5). The regression parameters calculated from the present series were comparable to those obtained from the literature (table 6). However, the influence of age, BMI and weight, although significant, was small, as shown by the overall low regression parameters. In contrast, the betweensubject variance was high, as shown by the high SD of all $P I$,max measurements (table 2).

Repetition of all $P \mathrm{I}$,max measurements tended to result in higher values of the second $P \mathrm{I}, \max$ (table 7). Accordingly, the mean difference between the two measurements was not zero but positively shifted (fig. 1). The CR of $P I$,max,0.1 was lower than those of $P$ I,peak, $P \mathrm{I}, \max , 0.5$ and $P \mathrm{I}, \max , 1.0$ (table 7). However, mean, SD and range were different for all $P \mathrm{I}, \max$

Table 4. - Peak maximal inspiratory mouth pressure ( $P$ I,peak $)$ and plateau maximal inspiratory mouth pressure sustained for $1.0 \mathrm{~s}$ $(P \mathrm{I}, \mathrm{max}, 1.0)$ measured at residual volume

\begin{tabular}{|c|c|c|c|c|c|c|}
\hline \multirow[t]{2}{*}{ Age group yrs } & \multicolumn{3}{|c|}{ Males } & \multicolumn{3}{|c|}{ Females } \\
\hline & Subjects $n$ & $P \mathrm{I}$, peak $\mathrm{kPa}$ & $P \mathrm{I}, \max , 1.0 \mathrm{kPa}$ & Subjects $n$ & $P \mathrm{I}$, peak $\mathrm{kPa}$ & $P \mathrm{I}, \max , 1.0 \mathrm{kPa}$ \\
\hline $10-19$ & 25 & $13.0(7.4)$ & $10.8(5.8)$ & 18 & $10.9(5.9)$ & $8.9(4.4)$ \\
\hline $20-29$ & 84 & $12.6(7.1)$ & $10.5(5.6)$ & 87 & $9.9(5.1)$ & $8.1(3.8)$ \\
\hline $30-39$ & 55 & $12.2(6.8)$ & $10.3(5.3)$ & 80 & $9.0(4.3)$ & $7.4(3.2)$ \\
\hline $40-49$ & 30 & $11.8(6.5)$ & $10.0(5.1)$ & 58 & 8.1 (3.6) & $6.7(2.6)$ \\
\hline $50-59$ & 13 & $11.4(6.1)$ & $9.7(4.9)$ & 27 & $7.2(2.8)$ & $6.0(2.0)$ \\
\hline $60-69$ & 14 & $11.0(5.8)$ & $9.4(4.6)$ & 21 & $6.4(2.1)$ & $5.3(1.5)$ \\
\hline$>70$ & 8 & $10.7(5.5)$ & $9.1(4.4)$ & 13 & $5.5(1.4)$ & $4.6(0.9)$ \\
\hline
\end{tabular}

Data are presented as 50 th percentile, i.e. median (5th percentile). 
Table 5.-Regression parameters for peak maximal inspiratory mouth pressure $(P l$, peak $)$ and plateau maximal inspiratory mouth pressure sustained for $1.0 \mathrm{~s}(P \mathrm{l}, \max , 1.0)$ in the final model

\begin{tabular}{|c|c|c|c|c|}
\hline & \multicolumn{2}{|c|}{ Males } & \multicolumn{2}{|c|}{ Females } \\
\hline & RV & FRC & RV & FRC \\
\hline Subjects $n$ & \multicolumn{2}{|c|}{229} & \multicolumn{2}{|c|}{304} \\
\hline$P$ I,peak & & & & \\
\hline Age yrs & $-0.05^{\#}$ & $-0.04 *$ & $-0.09 * * *$ & $-0.08 * * *$ \\
\hline Weight kg & $0.05^{*}$ & $0.05^{*}$ & $0.04 *$ & $0.05^{\#}$ \\
\hline $\mathrm{BMI} \mathrm{kg} \cdot \mathrm{m}^{-2}$ & $0.27 * *$ & $0.20 * *$ & $0.11 *$ & $0.14 * * *$ \\
\hline \multicolumn{5}{|l|}{ PI,max, 1.0} \\
\hline Age yrs & $-0.04 *$ & $-0.04 * *$ & $-0.08 * * *$ & $-0.07 * * *$ \\
\hline Weight $\mathrm{kg}$ & $0.06^{* *}$ & $0.05^{*}$ & $0.04^{* *}$ & $0.04^{\#}$ \\
\hline $\mathrm{BMI} \mathrm{kg} \cdot \mathrm{m}^{-2}$ & $0.24^{\#}$ & $0.18^{*}$ & $0.11 *$ & $0.13 * * *$ \\
\hline
\end{tabular}

RV: residual volume; FRC: functional residual capacity; BMI: body mass index. ${ }^{*}: \mathrm{p}<0.05 ; * *: \mathrm{p}<0.01{ }^{*}: \mathrm{p}<0.005 ;{ }^{* * *}: \mathrm{p}<0.001$.

measurements, with the lowest values found for PI,max,0.1. Therefore, it was decided to calculate the ratio of $\mathrm{CR}$ and the range of the second measurement $(\mathrm{CR} /$ range) in order to compare the reproducibility in terms of the different variance of the different PI,max measurements. Accordingly, PI,peak, $P$ I,max, 0.5 and $P$ I,max,1.0 were comparably reproducible (fig. 1), but $\mathrm{CR} /$ range was higher for $P \mathrm{I}$,max,0.1, indicating its lower reproducibility (table 7).

\section{Discussion}

$P$ I,peak has previously been reported to be significantly higher than PI,max,1.0 in small studies [7, 15], but most of the participants in the large study of ENRIGHT et al. [11] maintained close to their maximal pressure for $\geqslant 0.5 \mathrm{~s}$ during the $P \mathrm{I}, \max$ manoeuvre. In the present study, $P \mathrm{I}$,peak was significantly higher than $P \mathrm{I}, \max , 0.5$ and $P \mathrm{I}, \max , 1.0$, but $P \mathrm{I}, \max , 0.5$ was also significantly higher than PI,max,1.0, indicating that the choice of $P \mathrm{I}$,peak or plateau pressures significantly influences absolute values of $P I$,max.

Reproducibility, regression parameters and betweensubject variability were comparable for $P$ I,peak, $P$ I,max, 0.5 and $P \mathrm{I}, \max , 1.0$. Interestingly, $P \mathrm{I}$,peak, $P \mathrm{I}$,max, 0.5 and $P \mathrm{I}, \max , 1.0$ were highly correlated, indicating that $P \mathrm{I}$,peak and plateau pressures are reflected by each other. However, PI,peak are easier to calculate, since PI,peak can be determined directly from the pressure trace, whereas it is suggested that plateau pressures can be calculated from the area computed for the 1-s mean [1], and adequate software is needed for that purpose. In contrast, in the present study and most previous studies, plateau pressures were defined as pressures maintained for $\geqslant 1 \mathrm{~s}$, and different definitions might hinder comparison of plateau pressures from different studies. In addition, the 1-s mean pressure [1] seems to be very close to the plateau pressure sustained for $0.5 \mathrm{~s}$ in the present study. Furthermore, the $P$ I,peak is closely related to the short and sharp voluntary inspiratory manoeuvre during the sniff test [19], and normal values of $P \mathrm{I}, \max , 1.0$ are still not well defined

Table 6. - Regression parameters obtained from the literature for plateau maximal inspiratory mouth pressure ( $P$,max) sustained for $1.0 \mathrm{~s}^{\#}$ measured at residual volume

$\begin{array}{lllll}\text { First author [Ref.] } & \text { Year } & \text { PI,max units } & \text { Females } & \text { Males }\end{array}$

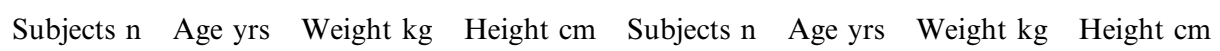

\begin{tabular}{|c|c|c|c|c|c|c|c|c|c|c|}
\hline BLACK [5] & 1969 & $\mathrm{cmH}_{2} \mathrm{O}$ & 60 & -0.51 & & & 60 & -0.55 & & \\
\hline WILSON [8] & 1984 & $\mathrm{cmH}_{2} \mathrm{O}$ & 87 & & & 0.71 & 48 & -1.03 & & \\
\hline VINCKEN [9] & 1987 & $\mathrm{cmH}_{2} \mathrm{O}$ & 60 & -0.67 & & & 46 & -0.82 & & \\
\hline ENRIGHT [11] ${ }^{\#}$ & 1994 & $\mathrm{cmH}_{2} \mathrm{O}$ & 1602 & -0.805 & 0.133 & & 1269 & -1.27 & 0.131 & \\
\hline HARIK-KHAN [13] & 1998 & $\mathrm{cmH}_{2} \mathrm{O}$ & 128 & -0.69 & 0.86 & -0.74 & 139 & -1.03 & 0.343 & \\
\hline MCCONNELL [15] & 1999 & $\mathrm{cmH}_{2} \mathrm{O}$ & 22 & -0.601 & 1.652 & 0.209 & 17 & -0.713 & -2.117 & 1.323 \\
\hline CARPENTER [14] & 1999 & $\mathrm{cmH}_{2} \mathrm{O}$ & 7197 & $-0.93^{\circledR}$ & ศ & & 5808 & $-1.20^{\top}$ & ฯ & ฯ \\
\hline HAUTMANN [16] & 2000 & $\mathrm{kPa}$ & 256 & -0.024 & & & 248 & $-0.051^{+}$ & + & + \\
\hline Present study & 2003 & $\mathrm{kPa}$ & 304 & $-0.08^{+}$ & $0.04^{+}$ & + & 229 & $-0.04^{+}$ & $0.06^{+}$ & + \\
\hline
\end{tabular}

\#: peak maximal inspiratory mouth pressure measured in [11]; ${ }^{\top}$ : regression parameter listed only for age; for different prediction equations for selected regression models, see [14]; ${ }^{+}$: body mass index was also a significant predictor or was included in the prediction equation. $1 \mathrm{cmH}_{2} \mathrm{O}=0.098 \mathrm{kPa}$.

Table 7. - Repeated measurement of maximal inspiratory mouth pressure $(P I, \max )$ and the coefficient of repeatability $(\mathrm{CR})$ at residual volume $(\mathrm{RV})$ and functional residual capacity (FRC)

\begin{tabular}{|c|c|c|c|c|c|c|}
\hline & $P \mathrm{I}, \max 1 \mathrm{kPa}$ & $P \mathrm{I}, \max 2 \mathrm{kPa}$ & Mean kPa & Difference $\mathrm{kPa}$ & $\mathrm{CR}^{\#} \mathrm{kPa}$ & $\mathrm{CR} /$ range $\%$ \\
\hline \multicolumn{7}{|l|}{ RV } \\
\hline$P \mathrm{I}$, peak & $11.83 \pm 3.73$ & $12.64 \pm 3.99$ & $12.24 \pm 3.72$ & $0.80 \pm 2.12$ & 4.49 & 25.18 \\
\hline$P \mathrm{I}, \max , 0.5$ & $10.61 \pm 3.79$ & $11.47 \pm 3.93$ & $11.04 \pm 3.70$ & $0.86 \pm 2.21$ & 4.70 & 27.35 \\
\hline$P \mathrm{I}, \max , 1.0$ & $9.74 \pm 3.79$ & $10.49 \pm 4.05$ & $10.11 \pm 3.76$ & $0.75 \pm 2.23$ & 4.67 & 26.19 \\
\hline$P I, \max , 0.1$ & $5.30 \pm 2.60$ & $5.82 \pm 2.48$ & $5.56 \pm 2.43$ & $0.52 \pm 1.48$ & 3.11 & 30.07 \\
\hline \multicolumn{7}{|l|}{ FRC } \\
\hline$P$ I,peak & $10.36 \pm 3.59$ & $10.73 \pm 3.61$ & $10.54 \pm 3.46$ & $0.37 \pm 2.00$ & 4.02 & 27.16 \\
\hline$P I, \max , 0.5$ & $9.57 \pm 3.53$ & $10.06 \pm 3.68$ & $9.82 \pm 3.49$ & $0.49 \pm 1.80$ & 3.07 & 24.93 \\
\hline$P \mathrm{I}, \max , 1.0$ & $8.74 \pm 3.52$ & $9.29 \pm 3.68$ & $9.02 \pm 3.51$ & $0.55 \pm 1.60$ & 3.35 & 23.15 \\
\hline$P I, \max , 0.1$ & $3.21 \pm 1.89$ & $3.83 \pm 2.00$ & $3.52 \pm 1.84$ & $0.62 \pm 1.30$ & 2.86 & 36.34 \\
\hline
\end{tabular}

PI,peak: peak maximal inspiratory mouth pressure; $P \mathrm{I}, \max , 0.5$ : plateau maximal inspiratory mouth pressure sustained for $0.5 \mathrm{~s} ; P \mathrm{I}, \mathrm{max}, 1.0$ : plateau maximal inspiratory mouth pressure sustained for $1.0 \mathrm{~s}$; $P$ I,max, 0.1 : maximal inspiratory mouth pressure $100 \mathrm{~ms}$ after start of maximal inspiratory manoeuvre; $\mathrm{n}=50$. \#: see [18]; ${ }^{\natural}$ : of the second measurement of $P$ I,max. 

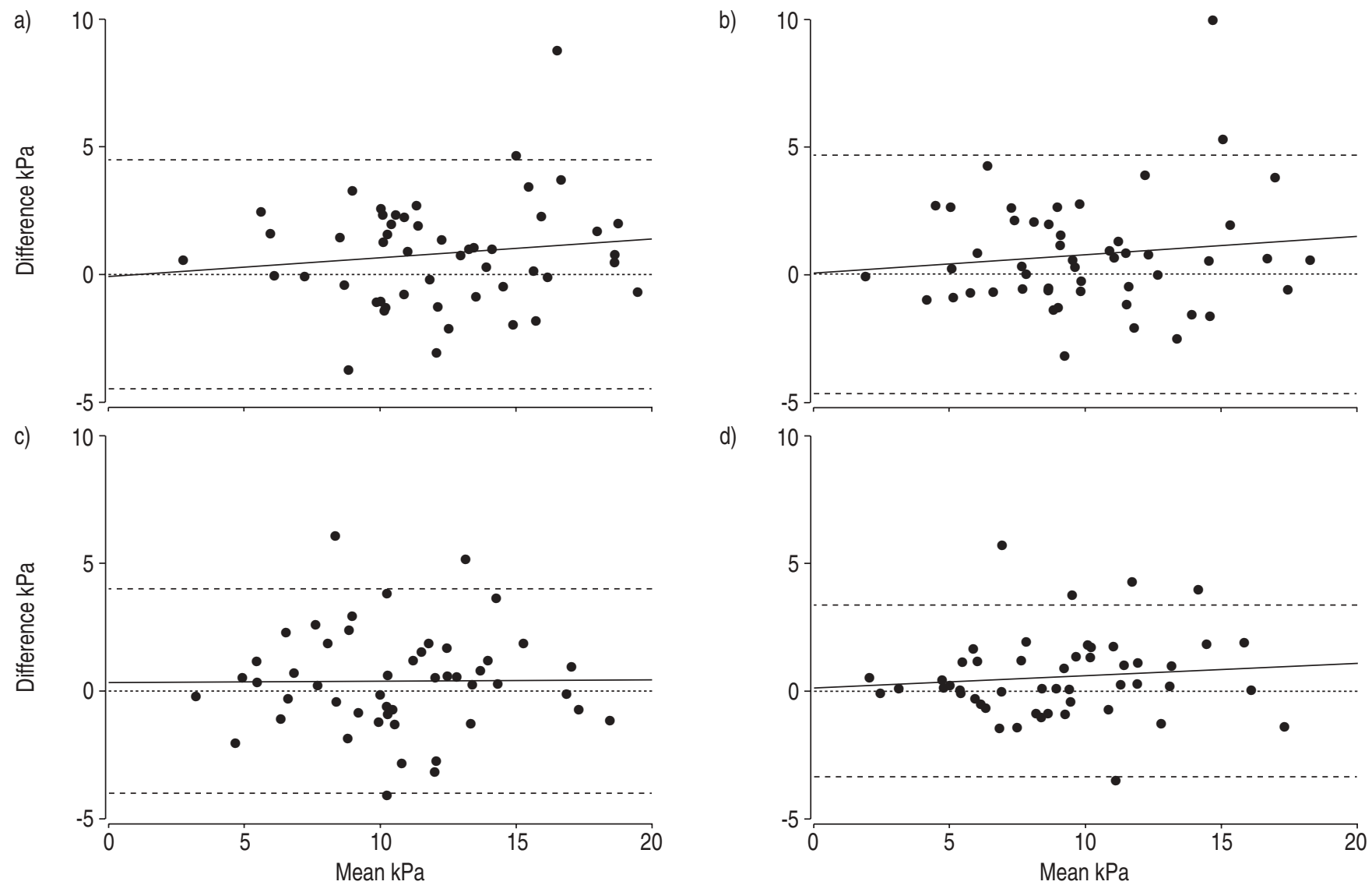

Fig. 1. - Repeatability of a) peak maximal inspiratory mouth pressure ( $P_{\mathrm{I}}$,peak $)$ measured at residual volume (RV), b) plateau maximal inspiratory

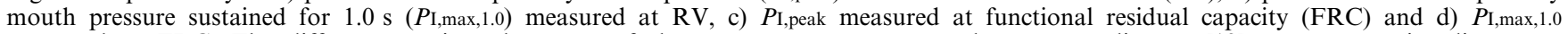
measured at FRC. The difference against the mean of the two measurements are shown, according to [18]. — : regression line; -----: reproducibility limits $(2 \mathrm{SD}) ; \cdots \cdots$ : zero reference $(n=50)$.

due to the wide range of reference values summarised in the ATS/ERS protocol [1]. Therefore, the present authors would suggest that $P$ I,peak are at least comparably useful to plateau pressures for determining inspiratory muscle strength, as has recently been announced in German guidelines on respiratory muscle testing [20].

$P$ I,max, 0.1 , determined from the mouth occlusion pressure/ time curve $100 \mathrm{~ms}$ after the start of inspiration during maximal voluntary inspiratory effort, has been introduced as a factor representative of $P I$, max $[21,22]$. As expected, $P \mathrm{I}, \mathrm{max}, 0.1$ is significantly lower than $P \mathrm{I}$,peak and plateau pressures, since it is known that the highest values of $P \mathrm{I}$,max are achieved 300-500 ms after the onset of inspiration [22]. In the present study, $P$ I,max,0.1 was less reproducible and more weakly correlated compared to $P \mathrm{I}$,peak or plateau pressures. This was expected, since $P \mathrm{I}, \max , 0.1$ reflects the large interindividual variability in the velocity of voluntary inspiratory muscle contraction during $P \mathrm{I}, \max$ manoeuvres.

It is well known that $P \mathrm{I}$,max decreases as lung volume increases [1, 3, 4, 23, 24]. In the present study, all $P \mathrm{I}$,max obtained at RV were significantly higher than those obtained at FRC, and this is probably attributable to the additional elastic recoil of the lungs and chest wall. Changes in the length/tension relationship might also contribute to these differences. In contrast to current belief, the PI,max manoeuvres were relatively easily performed at RV and at FRC, and nearly all participants were able to maximise their inspiratory effort at FRC. Regression parameters and between-subject variability were comparable when $P \mathrm{I}$, max was measured at FRC and at RV. Therefore, there was no clear advantage of FRC or RV as regards normal values.
However, the lung volume from which the inspiratory effort is performed is an important variable in determining absolute $P \mathrm{I}$, max values, as shown here and elsewhere [1], and underestimation of $P \mathrm{I}$,max can result from incomplete exhalation to $\mathrm{RV}$ if the corresponding normal values are based on $\mathrm{RV}$. In addition, repeated PI, max manoeuvres at FRC might be less tiring than those at RV, particularly in severe chronic obstructive pulmonary disease patients.

In agreement with the majority of previous studies, age was negatively predictive and weight and BMI were positively predictive for $P \mathrm{I}$, max $[5,8,9,11,13-16]$. However, despite significance, regression parameters were low, between-subject variability was high and only a small proportion of the total variance could be explained by demographic and anthropometric measures in both the present and previous studies [5, 8-11, 13-16]. However, $P \mathrm{I}, \max$ is a volitional test and hence can be submaximal and, therefore, variable. In addition, differences in physiological variables such as thoracoabdominal configuration, differences in the study group and technical issues are suggested to be responsible, at least in part, for the high between-subject variability. Furthermore, the variation in inspiratory pressures has been attributed to the variation in diaphragm thickness [25]. Some published regression equations are even in disagreement, since, for example, height has been shown to be positively predictive $[8,14,15]$, negatively predictive $[13]$ and not predictive $[5,9$, $11,16]$ for $P \mathrm{I}, \max$, and the number of variables included in the final model of the regression equation following stepwise regression varies significantly $[5,8,9,11,13-16]$. Therefore, the present authors would suggest that the regression 
equations in the literature are of scientific value, but are also inconsistent and not useful in clinical practice. For this reason, the 5th percentiles of $P$ I,peak and plateau pressures related to age group have been presented (table 4), and can be used as a guide to the lower limit of the normal range. However, low PI,max do not necessarily indicate respiratory muscle weakness, but, interestingly, a sniff test and measurement of mouth pressures during magnetic stimulation of the phrenic nerves used in combination can reliably exclude global inspiratory muscle weakness in most patients with normal inspiratory strength who have been screened for a low $P I$ max [26]. The number of patients who need to undergo more invasive tests such as pressure assessment using balloon catheters can thereby be reduced [26]. This is desirable, as these tests are more expensive and time-consuming, often unpleasant for the patient and difficult to perform, and are therefore the reserve of the few centres with adequate expertise [26]. However, owing to the present stringent exclusion criteria, only a few individuals aged $>70$ yrs were included in this study, and only patients aged $<70$ yrs were included in the study of Hughes et al. [26]. Therefore, the present authors would suggest that more data are necessary in order to give recommendations for the assessment of inspiratory muscle strength in this age group.

In conclusion, measurement of maximal inspiratory mouth pressure, despite its limitations, is by far the most widely used test for the assessment of inspiratory muscle strength, since it has no adverse effects and is noninvasive and easy to perform. The influence of demographic and anthropometric factors on normal values, although significant, is low, and unexplained between-subject variability is high. Therefore, regression equations for predicting normal values relative to demographic and anthropometric factors are less useful in clinical practice compared to 5 th percentiles, which can indicate the lower limit of the normal range. Peak pressures have no disadvantage compared to plateau pressures and can be comparably used. In contrast, the maximal inspiratory mouth pressure $100 \mathrm{~ms}$ after the start of maximal inspiratory effort is less useful in the assessment of inspiratory muscle strength. Maximal inspiratory mouth pressure can be reliably measured at both functional residual capacity and residual volume. However, further studies including patients with respiratory failure are needed in order to give clear recommendations as to how maximal inspiratory mouth pressure should be measured.

Acknowledgements. The authors would like to thank J. Schulte Mönting (Dept of Medical Statistics and Biometry, University of Freiburg, Freiburg, Germany) for statistical advice and R. Merklein (ZANß), Oberthulba, Germany) for writing the software.

\section{References}

1. Anon. ATS/ERS Statement on respiratory muscle testing. Am J Respir Crit Care Med 2002; 166: 518-624.

2. Anon. ATS/ACCP Statement on cardiopulmonary exercise testing. Am J Respir Crit Care Med 2003; 167: 211-277.

3. Cook CD, Mead J, Orzalesi MM. Static volume-pressure characteristics of the respiratory system during maximal efforts. J Appl Physiol 1964; 19: 1016-1022.

4. Ringqvist T. The ventilatory capacity in healthy subjects. An analysis of causal factors with special reference to the respiratory forces. Scand J Clin Lab Invest 1966; 18: Suppl. $88,8-170$

5. Black LF, Hyatt RE. Maximal respiratory pressures: normal values and relationship to age and sex. Am Rev Respir Dis 1969; 99: 696-702.

6. Leech JA, Ghezzo H, Stevens D, Becklake MR. Respiratory pressures and function in young adults. Am Rev Respir Dis 1983; 128: 17-23.

7. Smyth RJ, Chapman KR, Rebuck AS. Maximal inspiratory and expiratory pressures in adolescents. Normal values. Chest 1984; 86: 568-572.

8. Wilson SH, Cooke NT, Edwards RH, Spiro SG. Predicted normal values for maximal respiratory pressures in Caucasian adults and children. Thorax 1984; 39: 535-538.

9. Vincken W, Ghezzo H, Cosio MG. Maximal static respiratory pressures in adults: normal values and their relationship to determinants of respiratory function. Bull Eur Physiopathol Respir 1987; 23: 435-439.

10. McElvaney G, Blackie S, Morrison NJ, Wilcox PG, Fairbarn MS, Pardy RL. Maximal static respiratory pressures in the normal elderly. Am Rev Respir Dis 1989; 139: 2772-2781.

11. Enright PL, Kronmal RA, Manolio TA, Schenker MB, Hyatt RE. Respiratory muscle strength in the elderly. Correlates and reference values. Am J Respir Crit Care Med 1994; 149: 430-438.

12. Karvonen J, Saarelainen S, Nieminen MM. Measurement of respiratory muscle forces based on maximal inspiratory and expiratory pressures. Respiration 1994; 61: 28-31.

13. Harik-Khan RI, Wise RA, Fozard JL. Determinants of maximal inspiratory pressure. The Baltimore Longitudinal Study of Aging. Am J Respir Crit Care Med 1998; 158: 14591464.

14. Carpenter MA, Tockman MS, Hutchinson RG, Davis CE, Heiss G. Demographic and anthropometric correlates of maximum inspiratory pressure: the Atherosclerosis Risk in Communities Study. Am J Respir Crit Care Med 1999; 159: 415-422.

15. McConnell AK, Copestake AJ. Maximum static respiratory pressures in healthy elderly men and women: issues of reproducibility and interpretation. Respiration 1999; 66: 251258.

16. Hautmann H, Hefele S, Schotten K, Huber RM. Maximal inspiratory mouth pressures (PIMAX) in healthy subjects what is the lower limit of normal? Respir Med 2000; 94: 689693.

17. Quanjer PH, Tammeling GJ, Cotes JE, Pedersen OF, Peslin $\mathrm{R}$, Yernault JC. Lung volumes and forced ventilatory flows. Eur Respir J 1993; 6: Suppl. 16, 5-40.

18. Bland JM, Altman DG. Statistical methods for assessing agreement between two methods of clinical measurement. Lancet 1986; 1: 307-310.

19. Wijkstra PJ, van der Mark TW, Boezen M, van Altena R, Postma DS, Koeter GH. Peak inspiratory mouth pressure in healthy subjects and in patients with COPD. Chest 1995; 107: 652-656.

20. Criée CP. Recommendations of the German Airway League (Deutsche Atemwegsliga) for the determination of inspiratory muscle function. Pneumologie 2003; 57: 98-100.

21. Criée CP. Analysis of inspiratory mouth pressures. Prax Klin Pneumol 1988; 42: Suppl. 2, 820-826.

22. Criée CP, Neuhaus KL, Kreuzer H. Mouth occlusion pressure in patients with chronic obstructive lung disease in stable state and in acute respiratory failure. Bull Eur Physiopathol Respir 1982; 18: Suppl. 4, 155-164.

23. Rohrer F. Der Zusammenhang der Atemkräfte und ihre Abhängigkeit vom Dehnungszustand der Atmungsorgane. Arch Ges Physiol 1916; 165: 419-444.

24. Rahn H, Otis AB, Chadwick LE, Fenn WO. The pressurevolume diagram of the thorax and lung. Am J Physiol 1946; 146: 161-178.

25. McCool FD, Conomos P, Benditt JO, Cohn D, Sherman $\mathrm{CB}$, Hoppin FG Jr. Maximal inspiratory pressures and dimensions of the diaphragm. Am J Respir Crit Care Med 1997; 155: 1329-1334.

26. Hughes PD, Polkey MI, Kyroussis D, Hamnegard CH, Moxham J, Green M. Measurement of sniff nasal and diaphragm twitch mouth pressure in patients. Thorax 1998; 53: $96-100$. 